\title{
Research of Data Fusion Method of Multi-Sensor Based on Correlation Coefficient of Confidence Distance
}

\author{
Xinhua Jiang, Heru Xue ${ }^{*}$, Chen Wang and Yanqing Zhou \\ College of Computer and Information Engineering, Inner Mongolia Agricultural University, Hohhot, 010018, P.R. \\ China
}

\begin{abstract}
Because of the sensor or environment problem, the multi-sensor detection data can result in data mutation for the individual sensor. To obtain consistent, stable and accurate tested target description, the paper adopts confidence distance matrix, distance correlation coefficient and p-value significant test to get The number of optimal fusion number of multi-sensor, and uses maximum likelihood estimation to study the multi-sensor data fusion. The results show that the algorithm mentioned by the paper is superior to the multi-sensor consistency data fusion means and is fairly practical.
\end{abstract}

Keyword: Confidence distance, correlation coefficient, maximum likelihood estimation, p-value test, support.

\section{INTRODUCTION}

The detecting accuracy of single sensor is difficult to improve [1], and the failure and error of sensor can cause a decline at the respect of accuracy in the whole system. So independent multi-sensor which has low precision is adopted to achieve the same target detection, and improve the effectiveness and robustness of the detection system. In multisensor detection process, because of various orientations which the senor located, the difference of sensor and the effect of random factors in practical environment, the target value measured by each senor will have deviation [2]. Then, to reduce the errors that happen probably in the system processing, there is a question how to consistently accurately describe the measured target in the detection system. It is an effective method for the measured target to adopt the data fusion to implement multi-sensor consistency description. The key of data fusion is judging the data obtained by the every sensor of system, determining the credibility of the data, so as to decide on which data of sensor to do fusion [3]. Finally, the optimal fusion result for many target detection is acquired.

Data fusion methods involve signal processing, estimation theory, uncertainty theory, pattern recognition and optimization technique and so on [4], in accordance with, using the principle of statistics.

From the principle of statistics, using the actual collected data from the every sensor to calculate the confidence distance matrix, and adopting threshold to get relation matrix, which measure the correlation degree among sensors [5-8]. The confidence matrix can obtained the support degree to determine the optimal number of sensors. That threshold using to judge the mutual support between sensors exists a lot of fuzziness, and chooses subjectively the threshold to lead to the inaccurately fusion results [9-11]. Once data loss or disturb, the fusion result is not stable $[12,13]$. Determining the confidence distance threshold, the paper employs the confidence distance correlation coefficient $p$-value significant test to examine the related degree between sensors, ensures the optimal fusion number of multi-sensor under different confidence levels, and uses the maximum likelihood estimation to compute the final fusion results. Through the analysis of the confidence interval length and the measured values of each sensor which include standard deviation, root mean square error and residual sum of square, the final fusion results is confirmed. The multi-sensor data fusion mean mentioned by the paper is more scientific basis and practice, have an effective and reliable fusion result. The calculation process is simpler.

\section{THE COMPUTATION OF CONFIDENCE DISTANCE AMONG MULTI-SENSOR MEASURED VALUE}

Suppose there are $\mathrm{n}$ sensors that measure the same parameter from different orientation. $X_{i}=\left\{x_{i 1}, x_{i 2}, \ldots, x_{i m}\right\}$ is the data of the $i^{\text {th }}$ sensor. $i$ can value $1,2, \ldots, n$, and $1,2, \ldots \mathrm{m}$ is the $\mathrm{m}$ values of the ith sensor. Due to the factors of the sensor, measurement environment and measurement system stability, the actual values are errors with the measurements. In the actual measurement, the effect of all kinds of random factors will result in the measurement result $X_{i}$ to Gauss distribution. So measuring expectation of the sensor i can be described by Gauss probability density function as $\mathrm{P}_{\mathrm{i}}(\mathrm{x})$.

$$
P_{i}\left(x \mid x_{i}\right)=\frac{1}{\sqrt{2 \pi} \delta_{i}} \exp \left(-\frac{1}{2}\left(\frac{x-x_{i}}{\delta_{i}}\right)^{2}\right)
$$

$\mathrm{i}$ can value $1,2, \ldots \mathrm{n} . \mathrm{n}$ is the number of sensors.

On the definition of statistics, confidence needs sample statistic average $\mu$, variance $\delta$ and confidence intervals $[\delta-\Delta$, $\delta+\Delta]$. For Gauss distribution $\mathrm{N}(\mu, \delta)$, the confidence level c 
$c=\int_{\mu+\Delta}^{\mu-\Delta} N(\mu, \delta) d x$

To reflect the support degree of the data value between sensor $i$ and $j$, paper [11] adopts the confidence distance to measure $\mathrm{d}_{\mathrm{ij}}$.

$d_{i j}=\int_{x_{i}}^{x_{j}} p_{i}\left(x \mid x_{i}\right) d x$

$d_{j i}=\int_{x_{j}}^{x_{i}} p_{j}\left(x \mid x_{j}\right) d x$

$\mathrm{d}_{\mathrm{ij}}$ is the data confidence distance measure of the $\mathrm{i}^{\text {th }}$ and $\mathrm{j}^{\text {th }}$ sensor. It reflects the mutual support degree of two sensors data. $d_{i j}$ can use the error function $\operatorname{erf}(\theta)$ to obtain directly.

$d_{i j}=\operatorname{erf}\left(\frac{x_{j}-x_{i}}{\sqrt{2} \delta_{i}}\right)$

$d_{j i}=\operatorname{erf}\left(\frac{x_{i}-x_{j}}{\sqrt{2} \delta_{j}}\right)$

For $\mathrm{n}$ sensors measure a target at the same time, the confidence measure distance $\mathrm{d}_{\mathrm{ij}}(\mathrm{i}, \mathrm{j}=1,2, \ldots, \mathrm{n})$ consists a multisensor confidence distance matrix $\mathrm{D}_{\mathrm{nn}}$.

$D_{n n}=\left[\begin{array}{cccc}d_{11} & d_{12} & \ldots & d_{1 n} \\ d_{21} & d_{22} & \ldots & d_{2 n} \\ \ldots & \ldots & \ldots & \ldots \\ d_{n 1} & d_{n 2} & \ldots & d_{n n}\end{array}\right]$

\section{CONFIRMATION OF OPTIMAL FUSION NUMBER OF MULTI-SENSOR}

Taking advantage of the confidence distance matrix solves the mutual support degree of each sensor. Generally, the $\mathrm{d}_{\mathrm{ij}}$ threshold value $\varepsilon$ is given through the experience or the previous measure result, and sets up:

$r_{i j}= \begin{cases}1, & d_{i j} \leq \varepsilon \\ 0, & d_{i j}>\varepsilon\end{cases}$

$r_{i j}$ stands for the mutual support of $i$ and $j$ sensor. If confidence distance $\mathrm{d}_{\mathrm{ij}}$ is less than $\varepsilon$, then $\mathrm{r}_{\mathrm{ij}}$ is equal to 1 is regarded as the good correlation of $\mathrm{i}^{\text {th }}$ and $\mathrm{j}^{\text {th }}$ sensor. It calls that the $\mathrm{i}^{\text {th }}$ sensor supports the $\mathrm{j}^{\text {th }}$. If $\mathrm{r}_{\mathrm{ij}}$ is equal to 0 , it considers that the $i^{\text {th }}$ sensor does not support the the $\mathrm{j}^{\text {th }}$. If $r_{i j}$ and $r_{j i}$ is equal 1 , the $i^{\text {th }}$ sensor and $j^{\text {th }}$ sensor is mutual support. If $r_{i j}$ is larger than a certain extent, the data is valid, or the data is invalid. When multi-sensor measure the same parameter, the effective sensor set is fusion set. The sensor number of fusion set is called the optimal fusion number. $\varepsilon$ will be inevitably influence the result of data fusion. The optimal fusion number reduces and the data loss if $\varepsilon$ chooses improperly. The different $\varepsilon$ leads to the different fusion results.

Form the computation of $d_{i j}$, the value of $d_{i j}$ is between -1 and 1 . That $\mathrm{d}_{\mathrm{ij}}$ absolute value is greater shows the measurement deviation between two sensors. otherwise, the smaller value means the similar measured value of two sensors. Those reflect that the support degree of two sensors is high.
Under the condition of sensor normal, the measured value can consistently reflect the real values of measured object. The confidence distance matrix D will not appear sharp peaks and fat tail. So the support degree of two sensors is determined through probability correlation coefficient or related index. The most common method for correlation coefficient is product moment method which is called Pearson correlation coefficient. It uses the rate of the product covariance and standard deviation of two variables to indicate the correlation. For i and $\mathrm{j}$ sensor, confidence distance correlation coefficient can be defined as follows:

$r_{i j}=\frac{\delta_{d_{i} d_{j}}^{2}}{\delta_{d_{i}} \delta_{d_{j}}}=\frac{\sum \sum\left(d_{i k}-\overline{d_{i}}\right)\left(d_{j k}-\overline{d_{j}}\right)}{\sqrt{\sum \sum\left(d_{i k}-\overline{d_{i}}\right)^{2} \sum \sum\left(d_{j k}-\overline{d_{j}}\right)^{2}}}$

$r_{i j}=\frac{\delta_{d_{i} d_{j}}^{2}}{\delta_{d_{i}} \delta_{d_{j}}}=\frac{m \sum d_{i} d_{j}-\sum d_{i} \sum d_{j}}{\sqrt{m \sum d_{i}^{2}-\left(\sum d_{i}\right)^{2}} * \sqrt{m \sum d_{j}^{2}-\left(\sum d_{j}\right)^{2}}}$

$\bar{d}_{i}$ is the confidence distance mean between i sensor and other sensors. $\mathrm{r}_{\mathrm{ij}}$ is between -1 and 1 . Confidence distance correlation coefficient for $n$ sensors can make up a $n * n$ matrix $\mathrm{R}_{\mathrm{nn}}$.

$$
R_{n n}=\left[\begin{array}{cccc}
r_{11} & r_{12} & \ldots & r_{1 n} \\
r_{21} & r_{22} & \ldots & r_{2 n} \\
\ldots & \ldots & \ldots & \ldots \\
r_{n 1} & r_{n 2} & \ldots & r_{n n}
\end{array}\right]
$$

Related significant degree between senor measurements can be determined through testing confidence distance correlation coefficient. The paper uses the specified and different confidence level to examine the correlation coefficient significant $p$-value test and ensure the optimal number of multisensor fusion by the correlation coefficient significance. Confidence distance correlation coefficient significant $\mathrm{p}$ value for $\mathrm{n}$ sensors makes a $\mathrm{n} * \mathrm{n}$ matrix $\mathrm{P}_{\mathrm{nn}}$.

$P_{n n}=\left[\begin{array}{cccc}p_{11} & p_{12} & \ldots & p_{1 n} \\ p_{21} & p_{22} & \ldots & p_{2 n} \\ \ldots & \ldots & \ldots & \ldots \\ p_{n 1} & p_{n 2} & \ldots & p_{n n}\end{array}\right]$

Matrix $P$ is the symmetric matrix. $p_{i j}$ is the correlation significance $p$-value of $i$ and $j$ sensors. When $p_{i j}$ is not less than $\alpha$, $i$ sensor is no significant correlation with $j$ sensor. When $p_{i j}$ is less than $\alpha$, i sensor is significant correlation with $\mathrm{j}$ sensor. The optimal fusion number is composed of related and significant sensor.

\section{MULTI-SENSOR DATA FUSION}

Suppose the optimal fusion number of $\mathrm{n}$ sensors is $K\{\mathrm{x} 1, \mathrm{x} 2, \mathrm{x} 3, \ldots, \mathrm{xL}\}$. Adopting maximum likelihood estimation method calculates the data fusion results for $\mathrm{L}$ sensors. The formula (12) is establishing a likelihood function.

$$
\mathrm{p}(\hat{E} \mid \theta)=\prod_{k=1}^{l} p\left(x_{k} \mid \theta\right)
$$


To get logarithmic likelihood function of formula (12):

$\mathrm{H}(\theta)=\ln p(\hat{E} \mid \theta)=\sum_{k=1}^{l} \ln p\left(x_{k} \mid \theta\right)$

According to the theory of maximum likelihood estimation, sensor fusion result $\theta$ should satisfy the equation (14):

$\nabla_{\theta} H(\theta)=\sum_{k=1}^{l} \nabla_{\theta} \ln p\left(x_{k} \mid \theta\right)=0$

To solve the equation (14) can get the follow value:

$\left\{\begin{array}{c}\hat{\mu}=\frac{1}{l} \sum_{k=1}^{l} x_{k} \\ \hat{\delta}^{2}=\frac{1}{l} \sum_{k=1}^{l}\left(x_{k}-\hat{\mu}\right)^{2}\end{array}\right.$

The final solution:

$\hat{\theta}=\frac{\sum_{k=1}^{l} \frac{x_{k}}{\delta_{k}}}{\sum_{k=1}^{l} \frac{1}{\delta_{k}}}$

Table 1. The monitoring temperature data of 10 sensors.

\begin{tabular}{|c|c|c|c|c|c|c|c|c|c|c|c|c|}
\hline Sensor & \multicolumn{9}{|c|}{ Monitoring Value $\mathbf{x}_{\mathbf{i}}$} & \multicolumn{2}{c|}{ Mean } & Variance \\
\hline \hline 1 & 30.8 & 30.9 & 30.5 & 30.6 & 31.3 & 29.8 & 29.5 & 31.7 & 31.9 & 31.8 & 30.88 & 0.6707 \\
\hline 2 & 30.9 & 30.8 & 30.7 & 30.1 & 30.0 & 29.9 & 29.8 & 29.3 & 31.2 & 31.3 & 30.40 & 0.4467 \\
\hline 3 & 28.7 & 29.0 & 23.0 & 29.5 & 29.4 & 30.1 & 30.2 & 28.8 & 28.9 & 30.6 & 29.42 & 0.4440 \\
\hline 4 & 28.8 & 28.7 & 28.8 & 29.0 & 30.0 & 31.2 & 30.7 & 29.8 & 29.9 & 30.2 & 29.71 & 0.7499 \\
\hline 5 & 30.3 & 30.3 & 31.7 & 30.5 & 31.0 & 29.8 & 29.9 & 30.2 & 30.3 & 30.6 & 30.46 & 0.3049 \\
\hline 6 & 27.5 & 29.5 & 27.3 & 27.6 & 27.3 & 28.4 & 28.6 & 29.4 & 29.5 & 29.0 & 28.41 & 0.8543 \\
\hline 7 & 28.5 & 28.6 & 29.0 & 28.9 & 28.1 & 27.9 & 28.3 & 28.5 & 30.6 & 30.1 & 28.85 & 0.7472 \\
\hline 8 & 27.6 & 27.4 & 27.9 & 27.8 & 28.0 & 27.7 & 28.2 & 28.5 & 27.9 & 28.0 & 29.7 & 0.0956 \\
\hline 9 & 25.3 & 25.6 & 25.9 & 26.1 & 27.2 & 28.3 & 27.5 & 27.9 & 27.9 & 30.2 & 27.17 & 2.2334 \\
\hline 10 & 28.3 & 29.1 & 29.3 & 29.5 & 29.4 & 29.9 & 29.3 & 29.1 & 28.6 & 28.9 & 29.14 & 0.2093 \\
\hline
\end{tabular}

$D=\left[\begin{array}{cccccccccc}0 & -0.0154 & -0.0235 & 0.0067 & -0.0255 & 0.0039 & 0.0035 & -0.0453 & 3.90 e-4 & -0.0619 \\ -0.0154 & 0 & -3.74 e-4 & 0.0259 & 0.0018 & 0.0240 & 0.0278 & -0.0618 & 0.0041 & -0.0455 \\ -0.0235 & -3.74 e-4 & 0 & -0.0128 & 0.0219 & 0.0391 & 0.0228 & -0.0917 & 0.0302 & -0.0153 \\ 0.0067 & 0.0259 & -0.0128 & 0 & 0.0460 & 0.0075 & -2.06 e-4 & -0.1111 & 0.0081 & -0.0520 \\ -0.0255 & 0.0018 & 0.0219 & 0.0460 & 0 & 0.0395 & 0.0486 & -0.0488 & 0.0072 & -0.0226 \\ 0.0039 & 0.0240 & 0.0391 & 0.0075 & 0.0395 & 0 & 0.0051 & -0.0764 & 0.0518 & 0.0693 \\ 0.0035 & 0.0278 & 0.0228 & -2.06 e-4 & 0.0486 & 0.0051 & 0 & -0.1101 & 0.0391 & 0.0285 \\ -0.0453 & -0.0618 & -0.0917 & -0.1111 & -0.0488 & -0.0764 & -0.1101 & 0 & 0.1563 & -0.0445 \\ 3.90 e-4 & 0.0041 & 0.0302 & 0.0081 & 0.0072 & 0.0518 & 0.0391 & 0.1563 & 0 & 0.0833 \\ -0.0619 & -0.0455 & -0.0153 & -0.0520 & -0.0226 & 0.0693 & 0.0285 & -0.0445 & 0.0833 & 0\end{array}\right]$

$\hat{\theta}$ is the fusion data result of the optimal fusion number $K\{\mathrm{x} 1, \mathrm{x} 2, \mathrm{x} 3, \ldots, \mathrm{xL}\}$.

\section{DATA FUSION EXPERIMENTS}

\subsection{Experimental Data Sources}

In this paper, the temperature of the experimental data collected from oat planting environment in a sunlight greenhouse, many temperatures and humidity of wireless sensors were distributed in greenhouse, and temperature and humidity data of each sensor is collected every 15 minutes.

The temperature experimental data in Table 1 were collected from the 10 temperature and humidity sensors distributed different locations in Greenhouse, and acquisition time was early April 10:00 to 12:30 in northern region.

From Table 1 it can be seen the variance of the $9^{\text {th }}$ sensor changes slightly larger than other from the column of variance of measured data, from the actual position of the sensor in the greenhouse, the $9^{\text {th }}$ sensor was in the area of less sunlight than other sensor.

\subsection{Data Fusion Experiment}

First, using the formula (5) and (6) to calculate and obtain the confidence measure value distance matrix $D$ of the 10 sensors, $\mathrm{D}$ is a symmetric matrix. 


$$
R=\left[\begin{array}{cccccccccc}
1 & 0.8477 & 0.5355 & 0.6160 & 0.6923 & -0.0554 & 0.2217 & -0.0656 & -0.6525 & 0.2555 \\
0.8477 & 1 & 0.7936 & 0.7684 & 0.9345 & 0.2564 & 0.5086 & -0.2953 & -0.7065 & 0.3550 \\
0.5355 & 0.7936 & 1 & 0.8123 & 0.7131 & 0.6490 & 0.8123 & 0.0019 & -0.6865 & 0.6599 \\
0.6160 & 0.7684 & 0.8123 & 1 & 0.5455 & 0.5421 & 0.8131 & -0.1130 & -0.9217 & 0.1571 \\
0.6923 & 0.9345 & 0.7131 & 0.5455 & 1 & 0.2380 & 0.3984 & -0.4076 & -0.4869 & 0.4032 \\
-0.0554 & 0.2564 & 0.6490 & 0.5421 & 0.2380 & 1 & 0.9284 & 0.1267 & -0.5921 & 0.3062 \\
0.2217 & 0.5086 & 0.8132 & 0.8131 & 0.3984 & 0.9284 & 1 & 0.0312 & -0.8021 & 0.2930 \\
-0.0656 & -0.2953 & 0.0019 & -0.1130 & -0.4076 & 0.1267 & 0.0312 & 1 & 0.0065 & 0.4423 \\
-0.6525 & -0.7065 & -0.6865 & -0.9217 & -0.4869 & -0.5921 & -0.8021 & 0.0065 & 1 & -0.0521 \\
0.2555 & 0.3550 & 0.6599 & 0.1571 & 0.4032 & 0.3062 & 0.2930 & 0.4423 & -0.0521 & 1
\end{array}\right]
$$

$$
P=\left[\begin{array}{cccccccccc}
1 & 0.0001 & 0.0201 & 0.0042 & 0.0042 & 0.5270 & 0.1348 & 0.6035 & 0.0008 & 0.5108 \\
0.0001 & 1 & 0.0017 & 0.0031 & 0.0000 & 0.2792 & 0.0582 & 0.4716 & 0.0079 & 0.3135 \\
0.0201 & 0.0017 & 1 & 0.0051 & 0.0040 & 0.0473 & 0.0068 & 0.8020 & 0.0486 & 0.0392 \\
0.0042 & 0.0031 & 0.0051 & 1 & 0.0426 & 0.0544 & 0.0013 & 0.5180 & 0.0004 & 0.7343 \\
0.0042 & 0.0000 & 0.0040 & 0.0426 & 1 & 0.3899 & 0.1574 & 0.5139 & 0.0754 & 0.1756 \\
0.5270 & 0.2792 & 0.0473 & 0.0544 & 0.3899 & 1 & 0.0001 & 0.7657 & 0.1434 & 0.4946 \\
0.1348 & 0.0582 & 0.0068 & 0.0013 & 0.1574 & 0.0001 & 1 & 0.9290 & 0.0188 & 0.5328 \\
0.6035 & 0.4716 & 0.8020 & 0.5180 & 0.5139 & 0.7657 & 0.9290 & 1 & 0.7175 & 0.0365 \\
0.0008 & 0.0079 & 0.0486 & 0.0004 & 0.0754 & 0.1434 & 0.0188 & 0.7175 & 1 & 0.9245 \\
0.5108 & 0.3135 & 0.0392 & 0.1756 & 0.1756 & 0.4946 & 0.5328 & 0.0365 & 0.9245 & 1
\end{array}\right]
$$

By the formula (9) to calculate the sensors confidence distance correlation coefficient matrix R.

The correlation coefficient matrix $\mathrm{R}$ is calculated according to the experimental data obtained by sampling, there must inevitably be error, in order to infer the existence of correlation between each of the sensors, the method of hypothesis testing used in the experiments to test significant of correlation coefficient. By calculating significant $p$-value of the correlation coefficient data of each sensor, we get a significant test matrix $\mathrm{P}$ of correlation coefficient of the 10 sensors, and matrix $\mathrm{P}$ is a $10 * 10$ symmetric.

Table 2. The number of optimal fusion of different confidence level.

\begin{tabular}{|c|c|c|}
\hline $\begin{array}{c}\text { Confidence } \\
\text { Level }\end{array}$ & $\begin{array}{c}\text { The Number of Optimal } \\
\text { Fusion }\end{array}$ & $\begin{array}{c}\text { Result of Data } \\
\text { Fusion }\end{array}$ \\
\hline \hline$\alpha=0.05$ & $\mathrm{x} 1, \mathrm{x} 2, \mathrm{x} 3, \mathrm{x} 4, \mathrm{x} 9$ & 29.516 \\
\hline$\alpha=0.025$ & $\mathrm{x} 1, \mathrm{x} 2, \mathrm{x} 3, \mathrm{x} 4$ & 30.1025 \\
\hline$\alpha=0.01$ & $\mathrm{x} 2, \mathrm{x} 4$ & 30.055 \\
\hline
\end{tabular}

Experiments selected the confidence level $\alpha$ 0.05, 0.025 and 0.01 , and got the number of optimal fusion through calculating significant correlation test, and used formula (16) to calculate fusion results of the data of different confidence level $\alpha$, the results shown in Table 2 .

\subsection{Analysis of Fusion Results}

To use formula (16) to calculate the fusion results of monitoring data at confidence level $0.05,0.025$ and 0.01 .

As shown in Table 3, the confidence level is $0.05,0.025$ and 0.01 , and the length of the confidence interval of fusion results are respectively $0.9,0.6876$ and 1.0644 , but the three confidence interval length difference is not very obvious under the confidence levels. The root mean square error and residual sum of squares are calculated and obtained by the fusion results in Table 2 and the 100 monitoring results of 10 sensors in Table 1 under different confidence level, from results of the root mean square error RMSE and the residual sum of squares RSS, we can get the RMSE and RSS values under $\alpha=0.05$ smaller than $\alpha=0.025$ and 0.01 , the distribution of each sensors monitoring data concentrated with respect to the fusion results of 29.516 under the level of 0.05 .

Table 3. Maximum likelihood fusion results.

\begin{tabular}{|c|c|c|c|c|c|}
\hline Confidence Level & Result of Data Fusion & Confidence Interval & Standard Deviation & $\begin{array}{c}\text { Root Mean Square } \\
\text { Error (RMSE) }\end{array}$ & $\begin{array}{c}\text { Residual Sum of } \\
\text { Squares (RSS) }\end{array}$ \\
\hline \hline$\alpha=0.05$ & 29.516 & {$[29.066,29.966]$} & 1.5832 & 1.3994 & 195.8368 \\
\hline$\alpha=0.025$ & 30.1025 & {$[29.7587,30.4463]$} & 0.9327 & 1.6227 & 263.3136 \\
\hline$\alpha=0.01$ & 30.055 & {$[29.5228,30.5872]$} & 0.8319 & 1.5978 & 255.2885 \\
\hline
\end{tabular}


Table 4. Fusion results of 10 sensors on different monitoring time points.

\begin{tabular}{|c|c|c|c|c|c|c|c|c|c|c|c|c|}
\hline \multirow{2}{*}{$\begin{array}{l}\text { Confidence } \\
\text { Level }\end{array}$} & \multicolumn{10}{|c|}{ Fusion Results of Each Sensors on $T$ time } & \multirow{2}{*}{ RMSE } & \multirow{2}{*}{ RSS } \\
\hline & T1 & $\mathbf{T 2}$ & T 3 & $\mathbf{T} 4$ & T 5 & T 6 & $\mathbf{T} 7$ & T 8 & T 9 & $\mathbf{T} 10$ & & \\
\hline$\alpha=0.05$ & 28.77 & 29.09 & 29.01 & 28.96 & 29.17 & 29.3 & 29.2 & 29.32 & 29.55 & 29.97 & 0.1347 & 1.8151 \\
\hline$\alpha=0.025$ & 28.77 & 29.09 & 29.01 & 28.96 & 29.17 & 29.3 & 29.2 & 29.32 & 29.55 & 29.97 & 0.2962 & 8.5628 \\
\hline$\alpha=0.01$ & 28.77 & 29.09 & 29.01 & 28.96 & 29.17 & 29.3 & 29.2 & 29.32 & 29.55 & 29.97 & 0.2786 & 7.7602 \\
\hline
\end{tabular}

In order to further prove the fusion result at the confidence level of $\alpha=0.05$ is more precise than the fusion results of the levels of 0.025 and 0.01 , in this paper, we calculated respectively the fusion results of monitoring data of 10 sensors on each monitoring time point $\mathrm{T}$ under level of 0.05 , 0.025 and 0.01 by using the maximum likelihood estimation, the fusion results as shown in Table 4.

The root mean square error RMSE and the residual sum of squares RSS in Table $\mathbf{4}$ are calculated by the fusion results with T1-T10 monitoring times and the corresponding fusion result in Table 2 under different confidence level.

Through the above analysis, we selected result 29.516 as the ultimate fusion result, which is calculated by monitoring data of 10 sensors in Table $\mathbf{1}$ under the confidence level $\alpha=$ 0.05 .

\section{CONCLUSION}

In the multi-sensor detection system, data fusion is the purpose of using some algorithms to process comprehensively information and data from multi-sensor, and get more reliable and effective conclusion than a single sensor. Multisensor data fusion method presented in this paper is based on the monitoring data of each sensor, and then calculated the confidence distance between the monitoring data of sensors, and then tested the significant of correlation degree of confidence distance by the p-value test, and determined the optimal number of fusion of multi-sensor. The algorithms used in this paper avoid the subjective factors in determining the threshold of the fusion of the sensors, and have more adequate scientific basis. To use the maximum likelihood estimation method at different confidence level to obtain the final fusion results, and using the confidence interval length, root mean square error (RMSE) and the residual sum of squares (RSS) under different confidence level and analyze the fusion results, and finally get the optimal fusion results. To some extent, by experiments the data fusion methods used in this paper is simple and effective, and practical.

\section{CONFLICT OF INTEREST}

The authors confirm that this article content has no conflict of interest.

\section{ACKNOWLEDGEMENTS}

This work was supported in part by a grant from The Natural Science Foundation of the Inner Mongolia Autonomous Region (2014MS0623), and National Natural Science Foundation of China (61461041), and Technology Innovation Team of Inner Mongolia Agricultural University (NDPYTD2010-9).

\section{REFERENCES}

[1] J. Feng, C. Hegao, "Data fusion in sensor model", Journal of Transducer Technology, vol. 18, no. 6, pp. 47-49, 1999.

[2] Y. Yongxu, C. Xuhui, "The application of fuzzy set theory in multi-sensors information fusion", Computer Application and Software, vol. 28, no. 11, pp. 122-124, 2011.

[3] Z. Yanlong, W. Junyong, "Overview of the multi-senor data fusion technology", Ship Electronic Engineering, vol. 224, no. 2, pp. 4144, 2013.

[4] L. Jueshi, J. Jing and C. Huaxi, "An algorithom of multi-sensor information fusion based on the combination of rough set and neural network", Computer Application and Software, vol. 26, no. 2, pp. 14-16, 34, 2009.

[5] F. Zhengnan and S. Wenfu, "Analysis of correlation coefficient significance test”, Physical Experiment of College, vol. 22, no. 3, pp. 85-87, 90, 2009.

[6] W. Xu, C. Chang, Y.S. Hung, PCW Fung, "Asymptotic properties of order statistics correlation coefficient in the normal cases", IEEE Trans Signal Process, vol. 56, no. 6, pp. 2239-2248, 2008.

[7] X. Wei-chao, "A review on correlation coefficients", Journal of Guangdong University of Technology, vol. 29, no. 3, pp. 12-17, 2012.

[8] Z. Shun-zhong, W. Shu-mei, "Analysis on correlation coefficients matrix and multivariable linear correlation", College Mathematics, vol. 27, no. 1, pp. 195-198, 2011.

[9] H. Jiang and S. You-chao, "Application study of multi-sourced reliability data fusion method based on bayes theory", Aircraft DESIGN, vol. 32, no. 6, pp. 52-55, 2012.

[10] W. Xiao-jun, C. Qi-ying, C. Bao-xiang and L. Tong-ming, "Study on multisensor data fusion methods based on bayes estimation", Systems Engineering-Theory \& Practice, vol. 20, no. 7, pp. 45-48, 2000.

[11] C. Zengfu, "Mathematical method of multi-sensor data fusion", Journal of Mathematics in Practice and Theory, vol. 2, pp. 11-15, 1995.

[12] T. Guoping, "A robust method for the data fusion", Journal of Data Acquisition \& Processing, vol. 13, no. 1, pp. 85-87, 1998.

[13] L. Yi, L. Baichuan and L. Xue, "Multi-sensor data fusion based on multi-scale kalman filter", Journal of Chong Qing Jiao Tong University(Natural Science), vol. 31, no. 2, pp. 299-303, 2012.

Received: September 16, 2014

Revised: December 23, 2014

Accepted: December 31,2014

(C) Jiang et al.; Licensee Bentham Open.

This is an open access article licensed under the terms of the Creative Commons Attribution Non-Commercial License (http://creativecommons.org/licenses/by-nc/3.0/) which permits unrestricted, non-commercial use, distribution and reproduction in any medium, provided the work is properly cited. 